Article

\title{
Limitations and Constraints of Eddy-Current Loss Models for Interior Permanent-Magnet Motors with Fractional-Slot Concentrated Windings
}

\author{
Hui Zhang * and Oskar Wallmark \\ Department of Electric Power and Energy Systems, KTH Royal Institute of Technology, SE-100 44 Stockholm, \\ Sweden; owa@kth.se \\ * Correspondence: huizhang@kth.se; Tel.: +46-7-671-32-355
}

Academic Editor: K.T. Chau

Received: 2 January 2017; Accepted: 11 March 2017; Published: 16 March 2017

\begin{abstract}
This paper analyzes and compares models for predicting average magnet losses in interior permanent-magnet motors with fractional-slot concentrated windings due to harmonics in the armature reaction (assuming sinusoidal phase currents). Particularly, loss models adopting different formulations and solutions to the Helmholtz equation to solve for the eddy currents are compared to a simpler model relying on an assumed eddy-current distribution. Boundaries in terms of magnet dimensions and angular frequency are identified (numerically and using an identified approximate analytical expression) to aid the machine designer whether the more simple loss model is applicable or not. The assumption of a uniform flux-density variation (used in the loss models) is also investigated for the case of V-shaped and straight interior permanent magnets. Finally, predicted volumetric loss densities are exemplified for combinations of slot and pole numbers common in automotive applications.
\end{abstract}

Keywords: automotive applications; concentrated windings; eddy current losses; fractional-slot windings; interior permanent-magnet motors

\section{Introduction}

In interior permanent-magnet motors (IPMs), the permanent magnets (PMs) are embedded into the rotor. Compared to rotors with surface-mounted PMs, the resulting flux concentration effect in IPMs significantly reduces the induced PM eddy-current losses caused by the changing air-gap permeance due to the slot openings. Further, a not insignificant magnetic saliency can be realized, which contributes with a reluctance torque component and thereby increases the torque density. These are two main reasons why the IPM is a common machine topology when targeting automotive applications, with recent examples including [1-7].

A fractional-slot concentrated winding (FSCW) enables very short end-winding lengths and, thereby, potential improvements in terms of torque density. However, depending on the combination of the number of stator slots $Q_{s}$ and poles $p$, the resulting harmonic content in the air-gap magnetomotive force (MMF) caused by the stator currents can be substantial. During the last decade, efforts were put into identifying suitable combinations of $Q_{s}$ and $p$ where the impact of the stator MMF harmonics is as small as possible [8-10]. Particularly, for rotors with surface-mounted PMs, a number of models to quantify the induced eddy-current losses in the PMs based on the harmonic content in the stator MMF were developed [11-14]. Today, the combinations of $Q_{s}$ and $p$ with the lowest harmonic content have been identified, and FSCWs are adopted in automotive applications (see, e.g., [15] and the references in [16]). 
However, while the above loss models indeed are useful, they are most suitable for comparing the relative change in eddy-current losses for different combinations of $Q_{s}$ and $p$ rather than accurately predicting the losses for given PM dimensions. Particularly, the effect of segmentation of the magnets and the impact of the skin effect is challenging. Additionally, these models target surface-mounted PMs rather than IPMs (a typical FSCW-IPM motor with $Q_{s}=12$ slots and $p=8$ poles is depicted in Figure 1). Having a good approximation of the resulting PM losses is important for the machine designer since relatively small losses in the PMs can result in excessive temperatures due to the difficulty of transferring the resulting heat across the air gap.

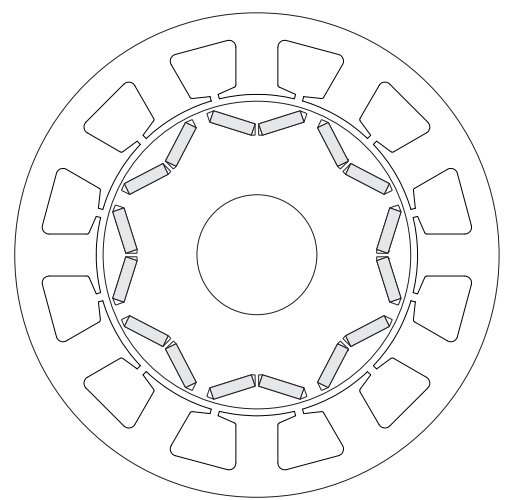

Figure 1. Sample fractional-slot concentrated winding (FSCW)-interior permanent-magnet motor (IPM) with $Q_{s}=12$ slots and $p=8$ poles.

Essentially, predicting the losses in the PMs can be done accurately using three-dimensional (3D) finite-element (FEM)-based simulations (as demonstrated in [17]). However, the approach must still be considered relatively time consuming, although efforts have been made to reduce the computation times [18]. Recently, the relatively simple analytical loss model in [19] was proposed considering FSCWs with surface-mounted PMs and including the effect of both axial and tangential segmentation of the PMs. This model uses the stator MMF harmonic content as input, but neglects the impact of the skin effect. A more advanced model, incorporating the effect of axial and tangential segmentation, as well as the skin effect, is adopted in [20] where analytical solutions of the Helmholtz equation with an imposed source term are adopted. A different model (also accounting for segmentation and the skin effect) is adopted in [21], where solutions to the Helmholtz equation with a prescribed boundary surface current are used.

\section{Contributions and Outline of the Paper}

An overall aim of this work is to provide a link between models describing the harmonic content in FSCWs due to the stator MMF with corresponding, sufficiently accurate PM eddy-current loss models for IPMs. Particularly, the loss models in [20] (here designated Model B) and [21] (here designated Model C), adopting solutions to different formulations of the Helmholtz equation to solve for the eddy currents, are compared to the considerably less complex loss model in [19] (here adapted to IPMs and designated Model A). It is demonstrated that Model B and Model C, though considerably different in terms of implementation complexity, predict very similar results (in good agreement with corresponding 3D-FEM simulations) for the PM dimension and rotor speed intervals typically found in automotive applications. Further, limits (in terms of frequency and PM dimensions) where Model A (the least complex loss model) is applicable are identified numerically and using an approximate analytical expression. This limit is not straight forward since the rectangular shape of the PM segments results in complex eddy-current reaction fields. Key concepts regarding FSCWs are included as an Appendix representing a complete description of how the uniform variation of the flux density in the PMs can be analytically predicted. The assumption of a uniform flux-density variation (used in the loss models) is also investigated for the case of V-shaped and straight interior permanent 
magnets. An extensive numerical evaluation using 3D-FEM-based simulations is carried out to verify model assumptions and and the conclusions made regarding whether each loss model is applicable or not. The comparison with 3D-FEM results represents a solid evaluation metric since 3D-FEM-based models of PM eddy-current losses previously have been demonstrated to yield good agreement with corresponding experimental results (see, e.g., $[17,21])$. Further, the extent of a corresponding experimental evaluation would be extremely expensive in order for a realization where a sufficiently large number of parameters (including PM, stator and rotor dimensions) would be varied.

The paper is outlined as follows. In Section 2, the loss models considered are briefly reviewed where the loss model in [19] (here designated Model A), but adapted to IPMs, is presented. In Section 3 (being the major part of this paper), model constraints when applied to IPM rotor geometries and the model limitations for Model A due to eddy-current reaction fields are identified. These constraints and limitations are also verified with presented 3D-FEM-based models. Finally, predicted magnet losses and temperature risk indicators are tabulated for combinations of $Q_{s}$ and $p$ that are commonly considered in automotive applications in Section 4, and concluding remarks are given in Section 5.

\section{Review of Eddy-Current Loss Models}

For axially short PMs and assuming a sinusoidal flux-density variation, the predicted volumetric loss density $p_{m}\left(\mathrm{~W} / \mathrm{m}^{3}\right)$ (presented in textbooks, e.g., [22,23]) can be expressed as:

$$
p_{m}=\frac{\sigma_{m} \omega_{v_{m}}^{2} l_{m}^{2} B_{v_{m}}^{2}}{24}
$$

where $\sigma_{m}$ is the conductivity of the PMs, $l_{m}$ the axial length of the PM and $\omega_{v_{m}}$ and $B_{v_{m}}$ are the angular frequency and magnitude of the imposed flux density, respectively.

However, the assumption of axially short PMs is not valid for practical axial segmentation lengths, and (1) cannot be directly applied. Generally, assuming constant $\mu_{r}$ and neglecting displacement currents (i.e., $\partial \mathbf{D} / d \partial t \approx 0$ ), the magnetic field in the PMs follows the Helmholtz equation, which can be expressed as:

$$
\nabla^{2} \overline{\mathbf{H}}=j \sigma_{m} \mu_{0} \mu_{r} \omega_{v_{m}} \overline{\mathbf{H}}
$$

Once a solution to (2) is determined, the current density (and associated losses) can be found from:

$$
\overline{\mathbf{J}}=\nabla \times \overline{\mathbf{H}} .
$$

In both (2) and (3), bold symbols denote vector fields, and the bar above denotes a phasor (complex) quantity. Solutions to (2) are used for loss Model B and Model C reviewed below.

\subsection{Model A: Assumed Eddy-Current Paths}

The loss model presented in [19] is developed for surface-mounted rotors where the air-gap flux-density harmonics passes over each permanent magnet segment depending on the rotor speed and wavelength of each harmonic. For IPMs, however, the flux concentration assumed in (A11) results only in a time-dependent variation of the flux density in the PMs. In [19], this corresponds to the case of an infinite wavelength where the assumed eddy-current paths are illustrated in Figure 2.

From Figure 2, the flux in the assumed eddy-current path $\phi$ can be expressed as:

$$
\phi=\int_{-y^{\prime}}^{y^{\prime}} \int_{-x^{\prime}}^{x^{\prime}} B_{v_{m}} \sin \left(\omega_{v_{m}} t\right) d x d y .
$$

The losses in the specific eddy-current path $d P_{m}$ can then be expressed as: 


$$
d P_{m}=\frac{(\partial \phi / \partial t)^{2}}{R_{\mathrm{EC}}}
$$

where $R_{\mathrm{EC}}$ is the resistance of the eddy-current path found as:

$$
R_{\mathrm{EC}}=\frac{4 y^{\prime}}{\sigma_{m} h_{m} d x^{\prime}}+\frac{4 x^{\prime}}{\sigma_{m} h_{m} d y^{\prime}} .
$$

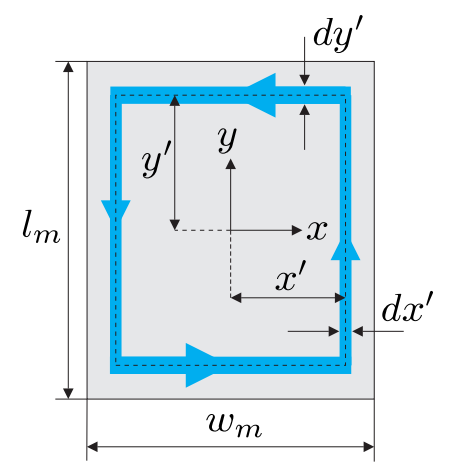

Figure 2. Assumed eddy-current paths adopted in Model A (redrawn from [19]).

The average magnet losses for an elementary current path $d P_{m \text {,ave }}$ (which is a function of $x^{\prime}$ and $\left.y^{\prime}\right)$ is now found by integrating (5) as:

$$
d P_{m, \text { ave }}=\frac{\omega_{v_{m}}}{2 \pi} \int_{0}^{2 \pi / \omega_{v_{m}}} d P_{m} d t
$$

Finally, an expression for the total average magnet losses for a magnet segment is now found as:

$$
P_{m}=\int_{0}^{w_{m} / 2} d P_{m, \text { ave }} d x^{\prime}=\frac{\sigma_{m} h_{m}\left(l_{m} w_{m}\right)^{3}\left(B_{v_{m}} \omega_{v_{m}}\right)^{2}}{32\left(l_{m}^{2}+w_{m}^{2}\right)}
$$

where $y^{\prime}=l_{m} x^{\prime} / w_{m}$ is used when evaluating the integral.

Remark 1. Note that for axially short PMs $\left(l_{m} \ll w_{m}\right)$, the volumetric loss density $p_{m}$ using (8) is found as:

$$
p_{m}=\frac{P_{m}}{h_{m} l_{m} w_{m}} \rightarrow \frac{\sigma_{m} \omega_{v_{m}}^{2} l_{m}^{2} B_{v_{m}}^{2}}{32}
$$

which is not in agreement with (1). From (9), it can, hence, be expected that Model A will underestimate the eddy current losses if the PMs are axially short.

Remark 2. Similar loss models also adopting prescribed eddy-current paths are presented in [24].

\subsection{Model B: Solving the Helmholtz Equation with the Imposed Source Term}

In [25], the Helmholtz equation is solved where the source field due to the armature reaction is added explicitly rather than as an imposed boundary condition. With this source term added, (2) becomes:

$$
\frac{\partial^{2} \bar{H}_{z}}{\partial x^{2}}+\frac{\partial^{2} \bar{H}_{z}}{\partial y^{2}}=j \sigma_{m} \mu_{0} \mu_{r} \omega_{v_{m}}\left(\bar{H}_{z}+\frac{B_{v_{m}}}{\mu_{0} \mu_{r}}\right)
$$


Similar solutions are reported in [20,26] where in [20], the solution is applied on two IPMs with distributed windings. The resulting average eddy-current losses for a magnet segment become [20]:

$$
\begin{aligned}
& P_{m}=\frac{32 \sigma_{m} \omega_{v_{m}}^{2} h_{m} l_{m} w_{m} B_{v_{m}}^{2}}{\pi^{2}} \sum_{n^{\prime}=1}^{\infty} \sum_{m^{\prime}=1}^{\infty}\left\{\frac{\text { num }}{\text { den }}\right\} \\
& \text { num }=\frac{1}{l_{m}^{2}\left(2 n^{\prime}-1\right)^{2}}+\frac{1}{w_{m}^{2}\left(2 m^{\prime}-1\right)^{2}} \\
& \text { den }=\pi^{4}\left[\frac{\left(2 n^{\prime}-1\right)^{2}}{w_{m}^{2}}+\frac{\left(2 m^{\prime}-1\right)^{2}}{l_{m}^{2}}\right]^{2}+\left(\frac{\mu_{0} \mu_{r} \sigma_{m} \omega_{v_{m}} h_{m}}{\delta+h_{m}}\right)^{2} \\
& \approx \pi^{4}\left[\frac{\left(2 n^{\prime}-1\right)^{2}}{w_{m}^{2}}+\frac{\left(2 m^{\prime}-1\right)^{2}}{l_{m}^{2}}\right]^{2}+\left(\mu_{0} \mu_{r} \sigma_{m} \omega_{v_{m}}\right)^{2}
\end{aligned}
$$

where $h_{m} \gg \delta$ was assumed in the approximation used in (13).

Remark 3. For axially short PMs; using (11), the volumetric loss density $p_{m}$ is found as:

$$
\begin{aligned}
p_{m} & =\frac{P_{m}}{h_{m} l_{m} w_{m}} \\
& =\frac{32 \sigma_{m} \omega_{v_{m}}^{2} l_{m}^{2} B_{v_{m}}^{2}}{\pi^{6}} \sum_{n^{\prime}=1}^{\infty} \sum_{m^{\prime}=1}^{\infty}\left\{\frac{1}{\left(2 n^{\prime}-1\right)^{2}\left(2 m^{\prime}-1\right)^{4}}\right\} .
\end{aligned}
$$

Now, the double sum in (14) equals $\pi^{6} / 768$ (obtained using Mathematica (Mathematica is a registered trademark of Wolfram Research, Inc., Champaign, IL, USA), which yields an expression for $p_{m}$ identical to (1).

\subsection{Model C: Solving the Helmholtz Equation Prescribing Boundary Surface Currents}

As is well known, the magnetization of a PM can be represented using a surface-current density with a magnitude corresponding to the remanent flux density divided by the permeability (see, e.g., [23]). Therefore, each flux density harmonic caused by the armature reaction can, approximately, be modeled assuming the surface current:

$$
J_{s}=\frac{B_{v_{m}}}{\mu_{0} \mu_{r}} \cos \left(j \omega_{v_{m}} t\right)
$$

on the outer boundaries of the magnet segment whose normals are in the $x y$-plane (the $x y$-coordinate system is depicted in Figure A1). Equation (2) simplifies to:

$$
\frac{\partial^{2} \bar{H}_{z}}{\partial x^{2}}+\frac{\partial^{2} \bar{H}_{z}}{\partial y^{2}}=j \sigma_{m} \mu_{0} \mu_{r} \omega_{v_{m}} \bar{H}_{z}
$$

with the boundary condition $\bar{H}_{z}=B_{v_{m}} /\left(\mu_{0} \mu_{r}\right)$ on the prescribed outer boundaries.

A general solution to (16) with the given boundary condition is presented in [21,27] where in [27], also the displacement currents are accounted for. From the solution $\bar{H}_{z}(x, y)$, the current density is then found as:

$$
\overline{\mathbf{J}}=\nabla \times \overline{\mathbf{H}}=\frac{\partial \bar{H}_{z}}{\partial y} \hat{x}-\frac{\partial \bar{H}_{z}}{\partial x} \hat{y}=\bar{J}_{x} \hat{x}+\bar{J}_{y} \hat{y}
$$

The resulting expression for the average eddy-current losses for a magnet segment becomes: 


$$
\begin{aligned}
& P_{m}= \frac{8 h_{m} w_{m} \omega_{v_{m}} B_{v_{m}}^{2}}{\pi^{2} \mu_{0} \mu_{r}} \sum_{p^{\prime}=1}^{\infty} \\
&\left\{\frac{\Im\{\alpha\} \sinh \left(\Re\{\alpha\} l_{m}\right)-\Re\{\alpha\} \sin \left(\Im\{\alpha\} l_{m}\right)}{m^{\prime 2}\left(\Re\{\alpha\}^{2}+\Im\{\alpha\}^{2}\right)\left[\cosh \left(\Re\{\alpha\} l_{m}\right)+\cos \left(\Im\{\alpha\} l_{m}\right)\right]}\right\} \\
& \quad+\frac{8 h_{m} l_{m} \omega_{v_{m}} B_{v_{m}}^{2}}{\pi^{2} \mu_{0} \mu_{r}} \sum_{q^{\prime}=1}^{\infty} \\
&\left\{\frac{\Im\{\beta\} \sinh \left(\Re\{\beta\} w_{m}\right)-\Re\{\beta\} \sin \left(\Im\{\beta\} w_{m}\right)}{n^{\prime 2}\left(\Re\{\beta\}^{2}+\Im\{\beta\}^{2}\right)\left[\cosh \left(\Re\{\beta\} w_{m}\right)+\cos \left(\Im\{\beta\} w_{m}\right)\right]}\right\}
\end{aligned}
$$

where $m^{\prime}=2 p^{\prime}-1, n^{\prime}=2 q^{\prime}-1$, and:

$$
\begin{aligned}
& \alpha=\sqrt{\left(\frac{m^{\prime} \pi}{w_{m}}\right)^{2}+j \sigma_{m} \mu_{0} \mu_{r} \omega_{v_{m}}} \\
& \beta=\sqrt{\left(\frac{n^{\prime} \pi}{l_{m}}\right)^{2}+j \sigma_{m} \mu_{0} \mu_{r} \omega_{v_{m}}}
\end{aligned}
$$

Remark 4. It is further shown in [27] that using (18), the resulting volumetric loss density $p_{m}$ for axially short PMs is identical to the classical expression (1).

\section{Analysis and Evaluation}

\subsection{Loss-Model Constraints When Applied to IPMs}

An analytical approach for approximating the flux-density variation $B_{m}\left(\theta_{r}\right)$ in the PMs as a function of rotor position $\theta_{r}$ is outlined in Section A.4 in the Appendix A. From $B_{m}\left(\theta_{r}\right)$, the corresponding harmonics (of order $v_{m}$ and with a magnitude $B_{v_{m}}$ ) can then be identified (in this paper, this identification has been done using the fft function in MATLAB (MATLAB is a registered trademark of The Mathworks Inc., Natick, MA, USA). However, if required, more accurate predictions of $B_{v_{m}}$ can rapidly be obtained using two-dimensional static FEM simulations.

The three eddy-current loss models reviewed in Section 2 all assume that the flux density variation in the PMs is uniform. For surface-mounted PMs, it is pointed out in [19] that such an assumption holds provided that the PM width $w_{m}$ is significantly lower than half of the wavelength $\lambda_{v}$ of the harmonic order of most interest $v$.

With the pole-cap coefficient $\alpha_{p}$ as defined in Figure A1, for IPMs, the corresponding condition can be expressed as $\alpha_{p} /(C p) \leq 1 / v$ where $C=1$ and $C=1 / 2$ for V-shaped and straight interior PMs, respectively. For many combinations of $Q_{s}$ and $p$, the harmonic order $v$ that dominates the PM losses is less than or equal to $p$ (i.e., $v \leq p$ ). Further, $\alpha_{p} \approx 3 / 4$ is not uncommon in order to realize a certain reluctance-torque component. Thereby, we obtain:

$$
\frac{3}{4} \leq \frac{C p}{v}
$$

and it can be concluded that for V-shaped PMs, a uniform flux-density variation in the PMs can often be assumed, whereas for straight interior PMs, this assumption is valid only if the harmonic order $v$ that dominates the PM losses fulfills $v \ll p$.

\subsection{Limits for Model A Due to Eddy-Current Reaction Fields}

As seen, the expressions for the volumetric loss density $p_{m}$ given by Model A, Model B and Model $\mathrm{C}$ are of increasing complexity. An interesting issue is therefore to determine the boundaries when the simplest model (Model A) is applicable. Since Model A does not incorporate the effect of eddy-current reaction fields, it may risk overestimating the eddy-current losses at higher frequencies. 
However, as pointed out in (9), Model A will underestimate the eddy-current losses if either $l_{m} \ll w_{m}$ or $w_{m} \ll l_{m}$ (where both Model B and Model C simplify to (1), as shown by (14) and in [27], respectively).

To compare Model B and Model C to Model A for typical PM dimensions $l_{m}$ and $w_{m}$, the relative errors $\varepsilon_{\mathrm{A} \mid \mathrm{B}}$ and $\varepsilon_{\mathrm{A} \mid \mathrm{C}}$ are therefore introduced as:

$$
\begin{aligned}
\varepsilon_{\mathrm{A} \mid \mathrm{B}} & =\frac{p_{m}(\text { Model } \mathrm{A})-p_{m}(\text { Model } \mathrm{B})}{p_{m}(\operatorname{Model} \mathrm{B})} \\
\varepsilon_{\mathrm{A} \mid \mathrm{C}} & =\frac{p_{m}(\text { Model } \mathrm{A})-p_{m}(\operatorname{Model} \mathrm{C})}{p_{m}(\operatorname{Model} \mathrm{C})} .
\end{aligned}
$$

In Figure 3, contours when $\varepsilon_{\mathrm{A} \mid \mathrm{B}}=0.2$ (representing a modest deviation) have been plotted for $\omega_{v_{m}} /(2 \pi)=300 \mathrm{~Hz}$ to $\omega_{v_{m}} /(2 \pi)=3000 \mathrm{~Hz}$ in steps of $300 \mathrm{~Hz}$ assuming $\mu_{r}=1.04$ and $\sigma_{m}=694 \mathrm{kS} / \mathrm{m}$ (typical values for PMs of the NdFeB-type). The regions representing $l_{m} \ll w_{m}$ or $w_{m} \ll l_{m}$ are patched as black in Figure 3. It is further found that the contours for $\varepsilon_{\mathrm{A} \mid \mathrm{C}}=0.2$ are essentially identical. Hence, Model B should predict very similar results to (the somewhat more complex) Model C. How Figure 3 (or its approximation Figure 4 (see below)) can be used by the machine designer to determine the validity of the different loss models is exemplified in Section 3.3.

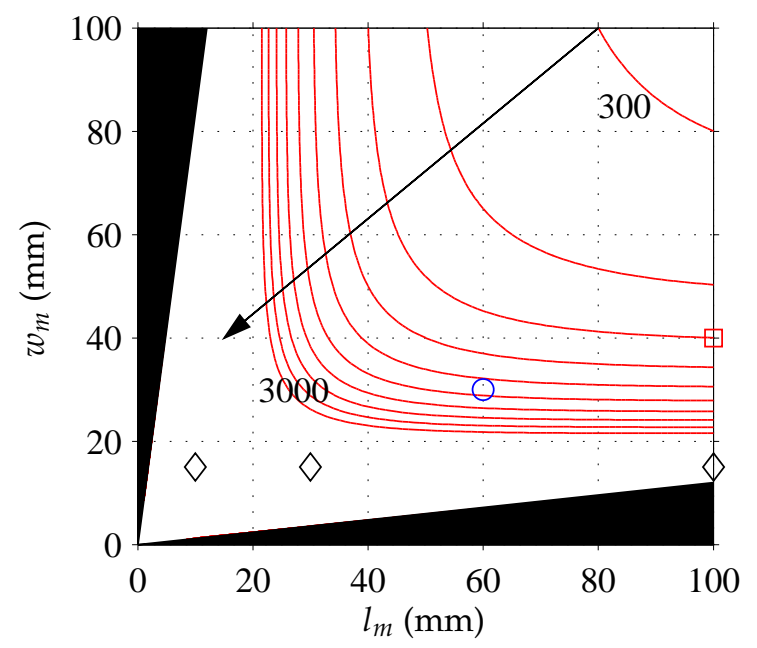

Figure 3. Contours corresponding to $\varepsilon_{\mathrm{A} \mid \mathrm{B}}=0.2$ (red curves) for $\omega_{v_{m}} /(2 \pi)=300 \mathrm{~Hz}$ to $\omega_{v_{m}} /(2 \pi)=$ $3000 \mathrm{~Hz}$ in steps of $300 \mathrm{~Hz}$ assuming $\mu_{r}=1.04$ and $\sigma_{m}=694 \mathrm{kS} / \mathrm{m}$. The arrow denotes the direction of increasing $\omega_{v_{m}}$. Note that the contours $\varepsilon_{\mathrm{A} \mid \mathrm{C}}=0.2$ are essentially identical.

Approximation of $\varepsilon_{\mathrm{A} \mid \mathrm{B}}$

Figure 3 can be useful for a machine designer since it provides boundaries in terms of magnet dimensions $l_{m}$ and $w_{m}$ and excitation frequencies $\omega_{v_{m}}$ when the simplest loss model (Model A) predicts the resulting eddy-current losses with sufficient accuracy. In [27], a number of approximations to (18) (Model C) are presented. However, when applying these approximation using typical PM dimensions $\left(l_{m}\right.$ and $\left.w_{m}\right)$ and a conductivity $\sigma_{m}$ valid for NdFeB-type PMs, it is found that none of the resulting approximations to $\varepsilon_{\mathrm{A} \mid \mathrm{C}}$ reproduce the contours in Figure 3 sufficiently accurately.

In order to investigate whether $\varepsilon_{\mathrm{A} \mid \mathrm{B}}$ can be approximated, the dimensional ratios $\xi$ and $\kappa$ are introduced as:

$$
\begin{gathered}
\xi=\frac{\max \left(l_{m}, w_{m}\right)}{\min \left(l_{m}, w_{m}\right)} \\
\kappa=\frac{\min \left(l_{m}, w_{m}\right)}{\delta_{\text {skin }}}
\end{gathered}
$$


where $\delta_{\text {skin }}=\sqrt{2 /\left(\sigma_{m} \mu_{0} \mu_{r} \omega_{v_{m}}\right)}$ is the classical expression for skin depth. Now, by considering only $n^{\prime}=m^{\prime}=1$ in $(11), \varepsilon_{\mathrm{A} \mid \mathrm{B}}$ can be approximated as:

$$
\varepsilon_{\mathrm{A} \mid \mathrm{B}} \approx\left[\frac{\pi^{2}}{256}\left(\frac{\xi^{2} \kappa^{2}}{1+\xi^{2}}\right)^{2}+\frac{\pi^{6}}{1024}\right]-1
$$

The contours for $\varepsilon_{\mathrm{A} \mid \mathrm{B}}=0.2$ using the approximation (26) are plotted in Figure 4. Comparing Figures 3 and 4, it can be seen that (26) represents the model prediction error reasonably well.

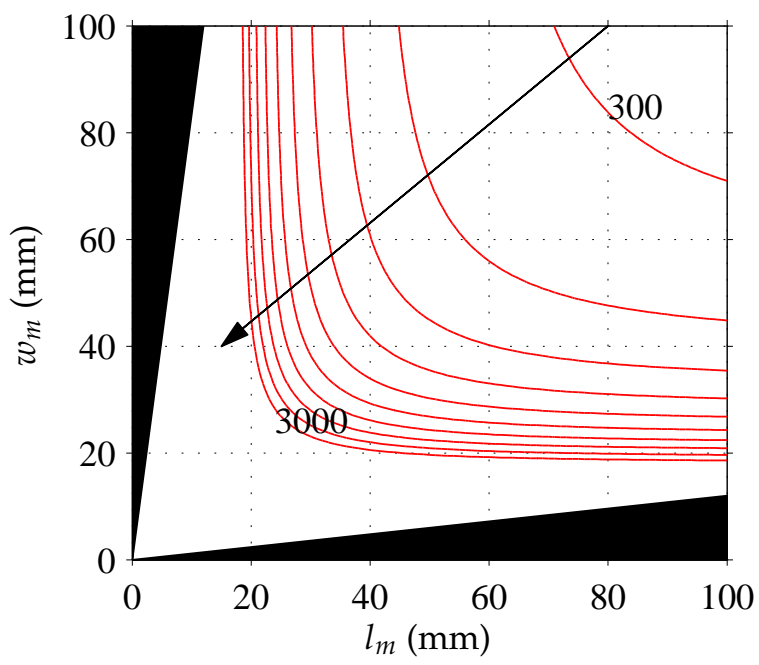

Figure 4. Contours corresponding to $\varepsilon_{\mathrm{A} \mid \mathrm{B}}=0.2$ (red curves) using the approximate formulation of $\varepsilon_{\mathrm{A} \mid \mathrm{B}}$ given by (26) for $\omega_{v_{m}} /(2 \pi)=300 \mathrm{~Hz}$ to $\omega_{v_{m}} /(2 \pi)=3000 \mathrm{~Hz}$ in steps of $300 \mathrm{~Hz}$ assuming $\mu_{r}=1.04$ and $\sigma_{m}=694 \mathrm{kS} / \mathrm{m}$. The arrow denotes the direction of increasing $\omega_{v_{m}}$.

\subsection{DFEM-Evaluation}

In order to verify the boundary constraints identified in Sections 3.1 and 3.2, comparisons with corresponding 3D-FEM-based models, implemented using JMAG (JMAG is a registered trademark of the JSOL Corporation, Tokyo, Japan), are now presented. In the 3D-FEM-based model, the eddy-current losses have been obtained by computing the average value of the Ohmic losses in a PM segment (caused by the resulting current density distribution).

A three-phase IPM with $Q_{s}=12$ slots and $p=8$ poles with additional key parameters reported in Table A1 in Appendix B is used initially for evaluation. The machine geometry is depicted in Figure 5a; the PM width is $w_{m}=15 \mathrm{~mm}$, and the axial length of the PM is varied so that $l_{m}=10,30$ and $100 \mathrm{~mm}$, respectively. The rotor speed is varied up to $9000 \mathrm{rpm}$.

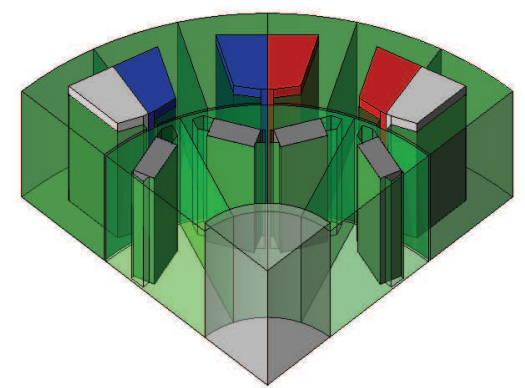

(a)

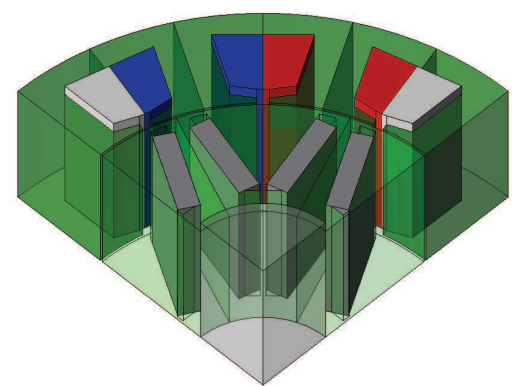

(b)

Figure 5. Cont. 


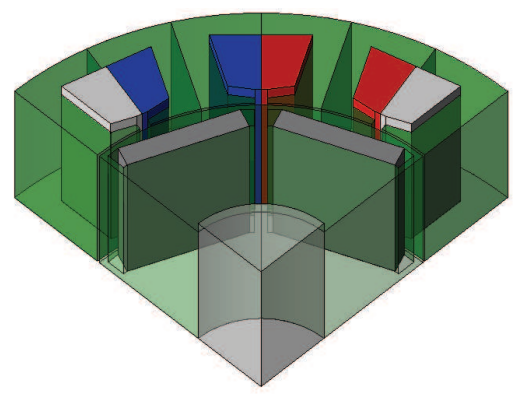

(c)

Figure 5. IPM 3D-FEM models: (a) V-shaped interior PMs $\left(w_{m}=15 \mathrm{~mm}\right)$; (b) V-shaped interior PMs $\left(w_{m}=30 \mathrm{~mm}\right)$; and $(\mathbf{c})$ Straight interior PMs $\left(w_{m}=40 \mathrm{~mm}\right)$.

A brief review of the fundamentals of the resulting harmonic content is provided in Appendix A. From (A1), the winding periodicity is found as $t_{\mathrm{per}}=4$. Therefore, from (A4), the harmonic orders that could be present in the air gap due to the armature reaction (assuming sinusoidal phase currents) are $v=4,8,12,16,20, \ldots$. However, from (A6), $k_{v}=0$ for harmonics that are multiples of 12 . The magnitude of the corresponding harmonics can be obtained using (A3)-(A9). Hence, in the air gap, the three first harmonic orders are $v=4,8$ and 16 . For the considered rotor structures, $\alpha_{p} \approx 0.77$ and using (A5) and (A12), the first harmonic order present in a PM is $v_{m}=12$ (higher order harmonics exist, but are of significantly lower magnitude and contribute only minorly to the total eddy-current losses). Hence, at $9000 \mathrm{rpm}, \omega_{v_{m}} /(2 \pi)=12 \cdot 9000 / 60=1800 \mathrm{~Hz}$.

Remark 5. Since the PMs are mounted in the interior of the rotor, the resulting eddy-current losses due to the slot-opening effect are negligible compared to the eddy-current losses due to the armature reaction.

\subsubsection{Negligible Eddy-Current Reaction Fields}

The considered three PM dimensions are indicated in Figure 3 as black diamonds $(\diamond)$. As seen, for $1800 \mathrm{~Hz}$, all three PM dimensions fall within the set of $l_{m}$ and $w_{m}$ for which Model A should provide an accurate prediction (i.e., a deviation of at most $20 \%$ ). This is verified in Figure 6, where it can be seen that Model A predicts similar results as Model B (red lines) and Model C (green lines) in V-shaped interior PMs $\left(w_{m}=15 \mathrm{~mm}\right)$ with different magnet lengths $l_{m}$ and also agrees with the corresponding 3D-FEM results.

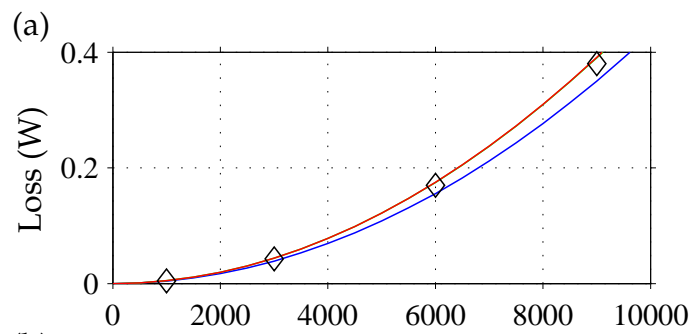

(b)

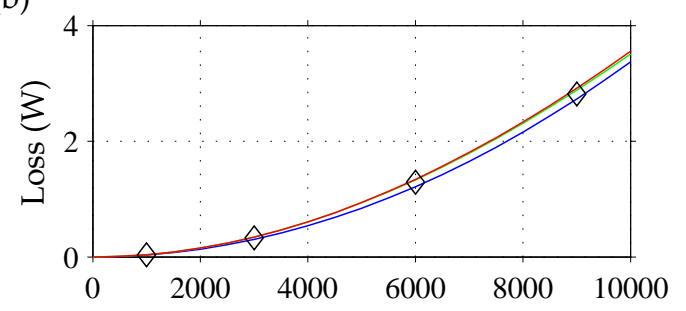

Figure 6. Cont. 


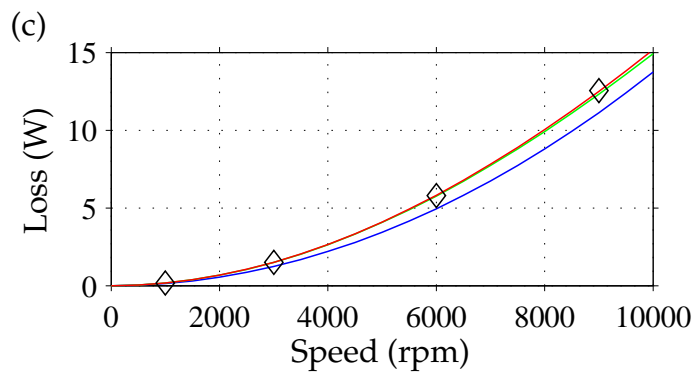

Figure 6. Predicted losses per magnet segment $P_{m}$ as a function of rotor speed and for different magnet lengths $l_{m}$ in V-shaped interior PMs $\left(w_{m}=15 \mathrm{~mm}\right.$ ) for Model A (blue line), Model B (red line), Model C (green line) and corresponding 3D-FEM results (diamonds " $\diamond$ "): (a) $l_{m}=10 \mathrm{~mm}$; (b) $l_{m}=30 \mathrm{~mm}$; and (c) $l_{m}=100 \mathrm{~mm}$.

\subsubsection{Non-Negligible Eddy-Current Reaction Fields}

With a wider magnet width $w_{m}=30 \mathrm{~mm}$ (see Figure 5b), it can be seen from Figure 3 (depicted as a blue circle (o)) that Model A will overestimate the eddy-current losses for frequencies above around $1700 \mathrm{~Hz}$ (corresponding to rotor speeds around $8500 \mathrm{rpm}$ ). This is verified in Figure 7 where the losses are substantially overestimated at speeds above (around) $8500 \mathrm{rpm}$. Compensating Model A as $P_{m} /\left(\varepsilon_{\mathrm{A} \mid \mathrm{B}}+1\right)$ using the approximation of $\varepsilon_{\mathrm{A} \mid \mathrm{B}}$ given by (26) yields a significantly better agreement with the corresponding 3D-FEM results. Model B and Model C predict similar results, both agreeing well with the corresponding 3D-FEM results at all rotor speeds considered.

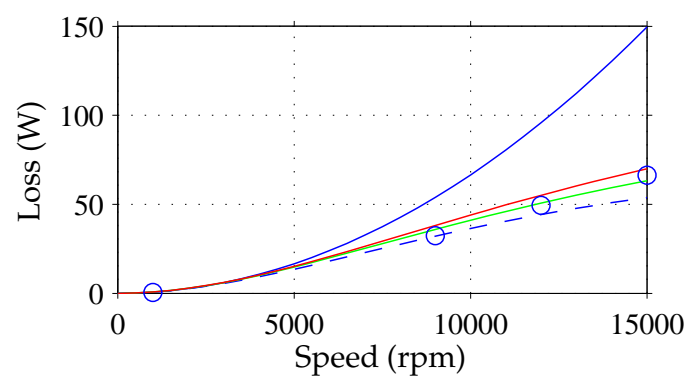

Figure 7. Predicted losses per magnet segment $P_{m}$ as a function of rotor speed and for magnet lengths $l_{m}=60 \mathrm{~mm}$ in V-shaped interior PMs $\left(w_{m}=30 \mathrm{~mm}\right)$ for Model A (blue lines), Model A, but compensated as $P_{m} /\left(\varepsilon_{\mathrm{A} \mid \mathrm{B}}+1\right)$ (dashed blue line), Model B (red line), Model C (green line) and corresponding 3D-FEM results (circles “०").

Remark 6. Since the PMs are buried very deep into the rotor structure, (A11) represents an overly simplistic approach for predicting the flux-density variation (while still being relatively uniform). Therefore, for this case only, the actual flux density $B_{m}\left(\theta_{r}\right)$ is extracted from static $2 D$-FEM models.

\subsubsection{Impact of Non-Uniform Flux-Density Variation}

Following the discussion in Section 3.1, the magnet width $w_{m}$ for the straight IPM depicted in Figure 5c (PM dimensions marked as the red square $(\square)$ in Figure 3 ) is wider than half of the dominant harmonic's wavelength $\lambda_{v}$. Therefore, the flux-density variation is not uniform, and the loss prediction accuracy for all three models worsens. This is verified in Figure 8 where neither of the models are in agreement with the corresponding 3D-FEM results. 


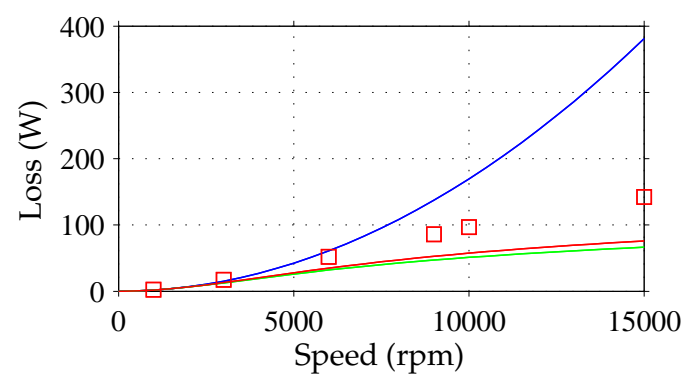

Figure 8. Predicted losses per magnet segment $P_{m}$ as a function of rotor speed for $l_{m}=60 \mathrm{~mm}$ for the straight IPM depicted in Figure $5 c\left(w_{m}=40 \mathrm{~mm}\right)$ for Model A (blue line), Model B (red line), Model C (green line) and corresponding 3D-FEM results (squares “ $\square$ ").

\subsection{Visualization of the Resulting Eddy-Current Distribution}

As is well known, the time-dependent currents densities found from the time-harmonic (complex) formulations used in Model B and Model C can be determined as:

$$
\begin{aligned}
& J_{x}(t)=\Re\left\{\bar{J}_{x}\right\} \cos \left(\omega_{v_{m}} t\right)-\Im\left\{\bar{J}_{x}\right\} \sin \left(\omega_{v_{m}} t\right) \\
& J_{y}(t)=\Re\left\{\bar{J}_{y}\right\} \cos \left(\omega_{v_{m}} t\right)-\Im\left\{\bar{J}_{y}\right\} \sin \left(\omega_{v_{m}} t\right) .
\end{aligned}
$$

Figure 9 shows the resulting eddy-current distributions in a PM segment of the IPM depicted in Figure $5 \mathrm{~b}$ for two different speeds (1000 and 15,000 rpm) with a PM segment length $l_{m}=60 \mathrm{~mm}$; predicted distributions using Model B (red contours) and Model C (green contours). First, it is noted that the resulting eddy-current distributions predicted by the two models are essentially equal. Hence, both models can be expected to predict similar eddy-current losses. Further, at $1000 \mathrm{rpm}$, the eddy current distribution is very similar to what is assumed in Model A (compare with Figure 2). However, at higher speeds, the eddy current paths during the transition between "clockwise" and "anti-clockwise" current paths becomes more complex with additional eddies close to corners. Corresponding 3D-FEM results are depicted in Figure 10, and as seen, the eddy-current distributions predicted with Model B and Model $\mathrm{C}$ are in good agreement with the 3D-FEM results.
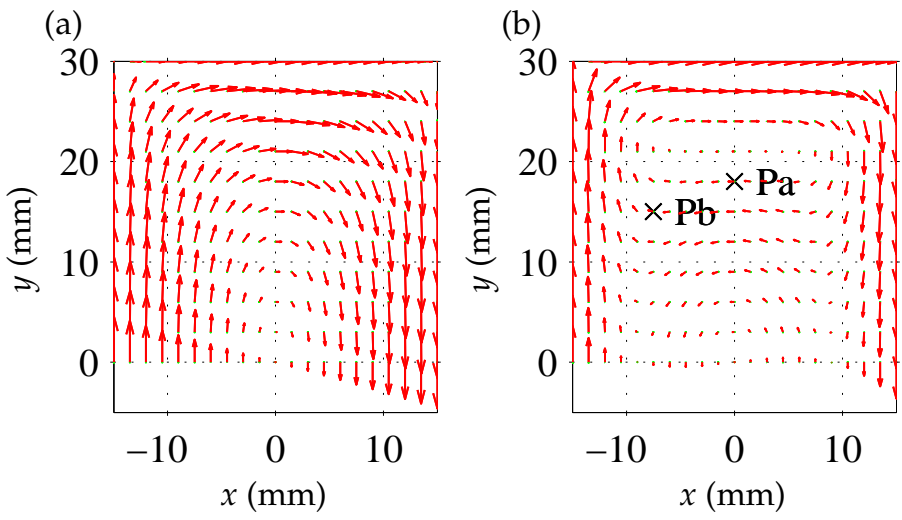

Figure 9. Resulting eddy current distribution using Model B (red contours) and Model C (green contours) evaluated at $\omega_{v_{m}} t=\pi / 4$ with a magnet length $l_{m}=60 \mathrm{~mm}$ at two different speeds: (a) $1000 \mathrm{rpm}$; (b) 15,000 rpm. Note that the green contours are almost completely covered by the red contours, which indicates that the two models predict essentially the same eddy current distribution. 

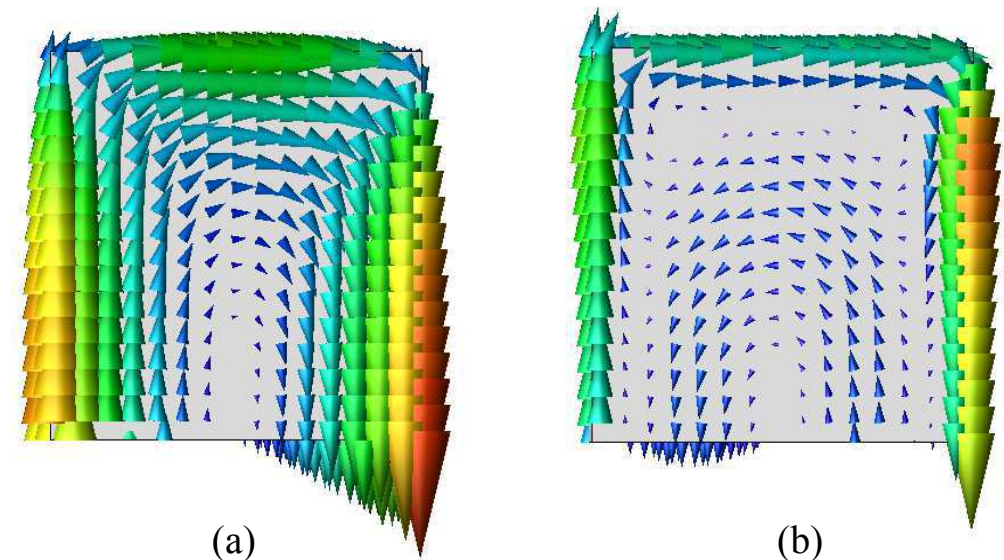

(b)

Figure 10. Resulting eddy current distribution from the 3D-FEM model evaluated at $\omega_{v_{m}} t=\pi / 4$ with a magnet length $l_{m}=60 \mathrm{~mm}$ at two different speeds: (a) $1000 \mathrm{rpm}$; (b) 15,000 rpm.

In Figure 11, the predicted current densities $|\mathbf{J}|=\sqrt{J_{x}^{2}+J_{y}^{2}}$ at 15,000 rpm in selected positions ' $\mathrm{Pa}$ ' and ' $\mathrm{Pb}$ ' (see Figure 9b) using Model A, Model B, Model C and the corresponding 3D-FEM results are reported. As seen, Model B and Model C are agreeing well with the 3D-FEM based results, whereas Model A overestimates the eddy-currents significantly.
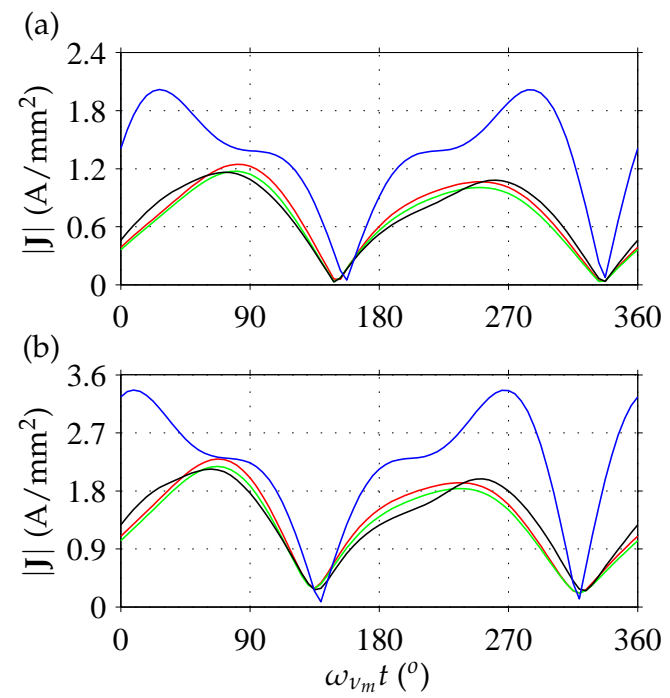

Figure 11. $|\mathbf{J}|$ evaluated at 15,000 rpm for Model A (blue lines), Model B (red lines), Model C (green lines) and 3D-FEM model (black lines) at selected points: (a) ‘ $\mathrm{Pa}^{\prime}$; (b) ‘ $\mathrm{Pb}^{\prime}$.

\section{PM Losses in Automotive Applications}

\subsection{Thermal Impact}

While Figure 3 or its approximation (26) provide necessary input on which loss model is suitable, the associated temperature increase in the PMs (due to the eddy currents) represents a key limiting factor for the machine designer. To investigate this thermal impact, a set of 3D-FEM thermal simulations of the machine described above is carried out at $9000 \mathrm{rpm}$ and rated current for different volumetric loss densities $p_{m}$ in the PMs. The implemented thermal model is similar to the 3D-FEM thermal model described in [28], and the intention is to determine approximately how different values of $p_{m}$ correspond to resulting magnet temperatures. The iron losses in the stator and rotor laminations are computed using the in-built loss model in JMAG. The winding, including the end-winding part, is 
impregnated in epoxy with an assumed effective thermal conductivity of $0.68 \mathrm{~W} /(\mathrm{m} \cdot \mathrm{K})$. The outer surface of the stator lamination is fixed to $60^{\circ} \mathrm{C}$ representing a water cooling jacket with a coolant temperature typical of what is found in automotive applications. A sample result from the 3D-FEM thermal simulation is depicted in Figure 12, and the resulting PM temperatures are reported in Figure 13. The results in Figure 13 will be used below in order to provide approximate intervals of $p_{m}$, which potentially can result in excessive PM temperatures in an automotive application.

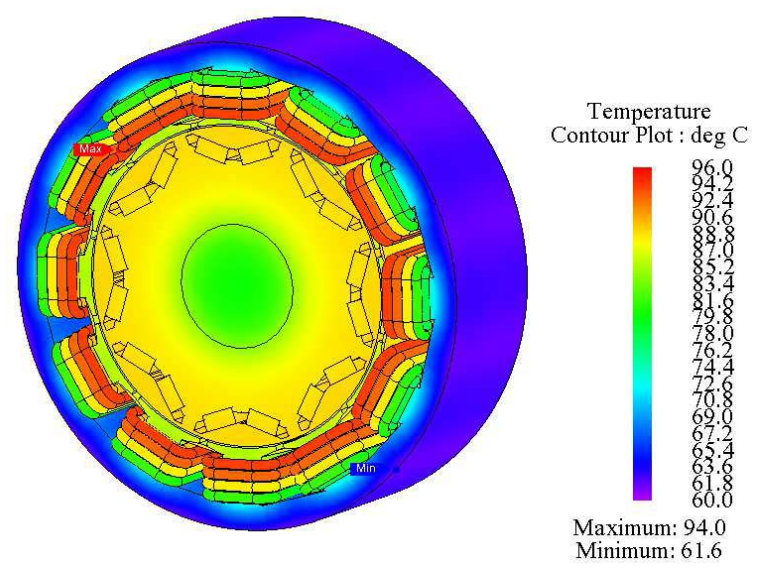

Figure 12. Implemented 3D-FEM thermal model with $p_{m}=0.1 \mathrm{~W} / \mathrm{cm}^{3}$ at rated current and $9000 \mathrm{rpm}$ (winding impregnation not shown).

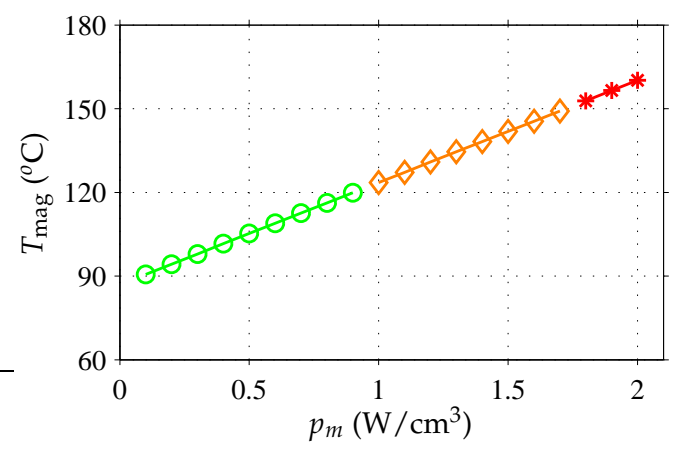

Figure 13. Resulting PM average temperature as a function of PM volumetric loss density $p_{m}$ at rated current and $9000 \mathrm{rpm}$. The considered low, medium and high temperature ranges have been indicated using green, orange and red colors, respectively.

\subsection{Losses for $p$ and $Q_{s}$ Common in Automotive Applications}

Now, resulting PM eddy-current losses for combinations of $p$ and $Q_{s}$ often considered in automotive applications are considered. The harmonic content from double-layer FSCWs is considered in this paper. For single-layer FSCWs, the harmonic content is described in $[8,12,14]$. The rotor radius $r_{r}$ and air-gap length $\delta$ are selected identical to what is reported in Table A1, and the main harmonic stator MMF (the ampere-turns and winding factor $k_{v=p / 2}$ product) is also kept the same, meaning that each resulting machine design results in a similar output torque (the harmonic content will be different, however, for each combination of $p$ and $Q_{s}$ ). The pole-cap coefficient is selected as $\alpha_{p}=3 / 4$ to yield a rotor saliency, and the PM height is selected as $h_{m}=5 \mathrm{~mm}$. Further, the PM width $w_{m}$ is selected so that the no-load flux density in the air gap is 0.75 T. For $p=8,10,12$ and 14 , this results in $w_{m}=14.2,11.3,9.5$ and $8.1 \mathrm{~mm}$. Resulting rotor geometries for $p=8$ and $p=14$ (with magnetic bridges and air pockets inserted) are depicted in Figure 14.

The resulting PM loss densities for $l_{m}=10 \mathrm{~mm}$ and $l_{m}=30 \mathrm{~mm}$ are reported in Tables 1 and 2, respectively. As seen, only a few combinations of $p$ and $Q_{s}$ result in sufficiently low eddy-current 
losses so that excessive PM temperatures are avoided. Furthermore, the increase of PM length from $l_{m}=10 \mathrm{~mm}$ to $l_{m}=30 \mathrm{~mm}$ rules out all but two combinations of $p$ and $Q_{s}$. Further, the loss densities reported in Tables 1 and 2 are predictions using Model C. However, using Figure 3 or its approximation (26), it can be determined that Model A provides sufficient accuracy (i.e., within a $20 \%$ deviation from Model B and Model C) for all cases considered in Tables 1 and 2.

(a)

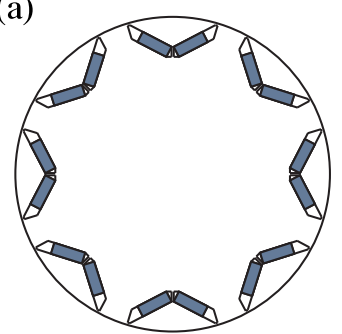

(b)

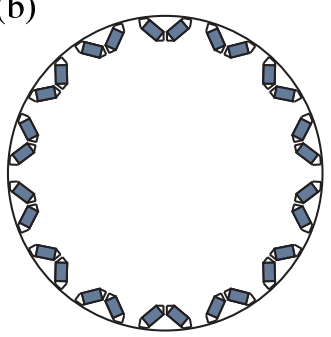

Figure 14. Rotor geometries (shaft excluded) for: (a) $p=8, \alpha_{p}=3 / 4, h_{m}=5 \mathrm{~mm}, w_{m}=14.2 \mathrm{~mm}$; (b) $p=14, \alpha_{p}=3 / 4, h_{m}=5 \mathrm{~mm}, w_{m}=8.1 \mathrm{~mm}$.

Table 1. $p_{m}\left(\mathrm{~W} / \mathrm{cm}^{3}\right)$ for $l_{m}=10 \mathrm{~mm}$ at $9000 \mathrm{rpm}$.

\begin{tabular}{|c|c|c|c|c|}
\hline$Q_{s} \backslash p$ & 8 & 10 & 12 & 14 \\
\hline 6 & 4.0 & 4.7 & N.F. & 4.1 \\
\hline 9 & N.F. & N.F. & 6.3 & N.F. \\
\hline 12 & 0.8 & 2.0 & N.F. & 6.2 \\
\hline 15 & N.F. & 1.0 & N.F. & N.F. \\
\hline 18 & 0.5 & 0.5 & 1.2 & 4.6 \\
\hline 21 & N.F & N.F. & N.F. & 1.3 \\
\hline 24 & \multirow[t]{3}{*}{$q_{s}=1$} & 5.6 & N.F. & 7.7 \\
\hline 27 & & N.F. & 0.8 & N.F. \\
\hline 30 & & $q_{s}=1$ & N.F. & 0.9 \\
\hline \multirow[t]{5}{*}{ N.F. } & \multirow{4}{*}{\multicolumn{4}{|c|}{$\begin{array}{c}\text { Not feasible/unbalanced winding } \\
\text { Distributed windings } \\
\text { Low losses } \\
\text { Medium losses }\end{array}$}} \\
\hline & & & & \\
\hline & & & & \\
\hline & & & & \\
\hline & \multicolumn{4}{|c|}{ High losses } \\
\hline
\end{tabular}

Table 2. $p_{m}\left(\mathrm{~W} / \mathrm{cm}^{3}\right)$ for $l_{m}=30 \mathrm{~mm}$ at $9000 \mathrm{rpm}$.

\begin{tabular}{|c|c|c|c|c|}
\hline$Q_{s} \backslash p$ & 8 & 10 & 12 & 14 \\
\hline 6 & 9.8 & 9.8 & N.F. & 6.7 \\
\hline 9 & N.F. & N.F. & 11.3 & N.F. \\
\hline 12 & 1.9 & 4.0 & N.F. & 9.1 \\
\hline 15 & N.F. & 2.0 & N.F. & N.F. \\
\hline 18 & 1.2 & 1.0 & 2.1 & 7.4 \\
\hline 21 & N.F & N.F. & N.F. & 2.2 \\
\hline 24 & \multirow[t]{3}{*}{$q_{s}=1$} & 11.5 & N.F. & 12.5 \\
\hline 27 & & N.F. & 1.4 & N.F. \\
\hline 30 & & $q_{s}=1$ & N.F. & 1.5 \\
\hline N.F. & \multirow{5}{*}{\multicolumn{4}{|c|}{$\begin{array}{c}\text { Not feasible/unbalanced winding } \\
\text { Distributed windings } \\
\text { Low losses } \\
\text { Medium losses } \\
\text { High losses }\end{array}$}} \\
\hline & & & & \\
\hline & & & & \\
\hline & & & & \\
\hline & & & & \\
\hline
\end{tabular}

\section{Conclusions}

In this paper, three models for predicting average magnet losses in IPMs with FSCWs due to induced eddy currents caused by the armature reaction (assuming sinusoidal phase currents) were 
analyzed and compared. Provided that the harmonic order $v$ that dominates the PM losses is less or equal to the number of poles $p$, it was found that that for V-shaped PMs, a uniform flux density variation in the PMs can typically be assumed. Boundaries in terms of PM dimensions and angular frequency were identified to aid the machine designer to decide whether the simplest loss model (Model A) is applicable or not. An approximate analytical expression to these boundaries was also identified. It was found that Model B and Model C, while relying on different formulations and solutions of the Helmholtz equation, provide very similar loss predictions in very good agreement with corresponding 3D-FEM-based simulations. Further, tables were provided with resulting volumetric loss densities $p_{m}$ for combinations of $Q_{s}$ and $p$ commonly used in automotive applications. Finally, by compiling results from previous publications, a complete description on how to analytically predict the magnitudes $B_{v_{m}}$ and corresponding harmonic orders $v_{m}$ for FSCW-IPMs was provided in the Appendix.

Acknowledgments: The financial support from the Swedish Hybrid Vehicle Center (SHC) and the STandUP for Energy research initiative is gratefully acknowledged.

Author Contributions: This paper is a result of the full collaboration of all of the authors. Hui Zhang and Oskar Wallmark have contributed by developing ideas and the analytical model. Hui Zhang built the FEM models and analyzed the data. Oskar Wallmark was responsible for the guidance and a number of key suggestions.

Conflicts of Interest: The authors declare no conflict of interest.

\section{Appendix A. FSCW Fundamentals}

How to predict the magnitudes $B_{v_{m}}$ and corresponding harmonic orders $v_{m}$ in FSCW-IPMs (compiling results from $[8,10,29,30]$ ) is, for completeness, described in this Appendix.

\section{Appendix A.1. Preliminaries}

The winding periodicity $t_{\text {per }}$ can be expressed as [8]:

$$
t_{\text {per }}=\operatorname{gcd}\left(Q_{s}, p / 2\right)
$$

where $Q_{s}$ is the number of stator slots, $p$ the number of poles and $\operatorname{gcd}(a, b)$ denotes the greatest common denominator for $a$ and $b$. Introducing $m_{\mathrm{ph}}$ as the (odd) number of phases, the (sinusoidal) current $i_{n}$ in phase $n, n=1,2, \ldots, m_{\mathrm{ph}}$, can be expressed as:

$$
i_{n}=\sqrt{2} I \cos \left[\frac{p}{2}\left(\theta_{r}-\alpha_{\mathrm{ph}}(n-1)-\varphi\right)\right] .
$$

Here, $\theta_{r}$ is the (mechanical) rotor position; $\varphi$ is the current phase angle angle; and $\alpha_{\mathrm{ph}}$ the angular displacement of the air-gap MMF distribution due to each adjacent phase $\left(\alpha_{\mathrm{ph}}=2 \pi /\left(m_{\mathrm{ph}} t_{\mathrm{per}}\right)\right)$.

\section{Appendix A.2. Air-Gap MMF Distribution Due to Stator Current}

Assuming that all coils belonging to each phase are series connected, the resulting air-gap MMF $F_{\delta}$ can be expressed as [31]:

$$
F_{\delta}=\sum_{v}\left\{\sum_{n=1}^{m_{\mathrm{ph}}} \frac{n_{s} Q_{s} k_{v} i_{n}}{\pi m_{\mathrm{ph}} v} \cos \left[v\left(\theta_{s}-\alpha_{\mathrm{ph}}(n-1)\right)\right]\right\}
$$

where $n_{s}$ is the number of turns per slot, $k_{v}$ the winding factor for harmonic $v$ and $\theta_{s}$ is an angle spanning the air-gap circumference (expressed in mechanical degrees). Equation (A3) is valid if magnetic saturation can be neglected, and it is derived assuming that the current in the stator slots can be represented as point-like sources. The harmonic orders $v$ that can be present in the air gap can be expressed as [8]: 


$$
v=\left\{\begin{array}{l}
(2 k-1) t_{\text {per }}, \quad \text { if } Q_{s} / t_{\text {per }} \text { is even } \\
k t_{\text {per }}, \quad \text { if } Q_{s} / t_{\text {per }} \text { is odd }
\end{array}\right.
$$

where $k$ is an integer. Now, $\operatorname{sgn}(v)$ is introduced to denote the direction of rotation of each MMF harmonic $v$ with respect to the rotor. Following from (A3) (see [29] for further details), $\operatorname{sgn}(v)$ can be expressed as:

$$
\operatorname{sgn}(v)= \begin{cases}+1, & \text { if rem }\left(\frac{p}{2}-v, m_{\mathrm{ph}} t_{\mathrm{per}}\right) \\ -1, & \text { if rem }\left(\frac{p}{2}+v, m_{\mathrm{ph}} t_{\mathrm{per}}\right)\end{cases}
$$

Note that (A5) is applicable only to harmonics $v$ that can be present in the air gap (defined by (A4)).

\section{Appendix A.3. Winding Factor for Harmonic $v$}

The winding factor for harmonic $v k_{v}$ can be expressed as [8,29]:

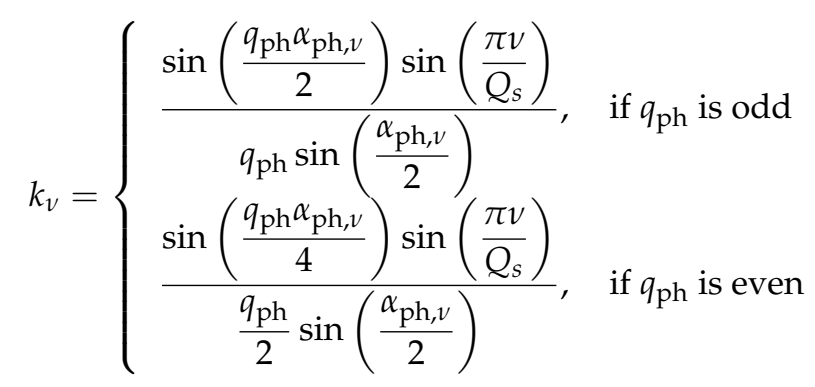

where the electrical angle between the electromotive force of two adjacent coils $\alpha_{\mathrm{ph}}$ and the number of spokes per phase in the star of slot $q_{\mathrm{ph}}$ are given as:

$$
\begin{aligned}
\alpha_{\mathrm{ph}, v} & =\pi-\frac{2 \pi v}{Q_{s}} \operatorname{round}\left(Q_{s} / p\right) \\
q_{\mathrm{ph}} & =Q_{s} /\left(m_{\mathrm{ph}} t_{\mathrm{per}}\right) .
\end{aligned}
$$

A sufficient condition for (A6) to be valid is [29]:

$$
\text { round }\left(Q_{s} / p\right)= \begin{cases}\frac{\operatorname{round}\left(\frac{Q_{s} q_{\mathrm{ph}}}{p}\right)}{q_{\mathrm{ph}}}, & \text { if } q_{\mathrm{ph}} \text { is odd } \\ \frac{\operatorname{round}\left(\frac{Q_{s} q_{\mathrm{ph}}}{2 p}\right)}{\frac{q_{\mathrm{ph}}}{2},} & \text { if } q_{\mathrm{ph}} \text { is even }\end{cases}
$$

If (A9) is not fulfilled, the winding factor can then be computed by numerically computing the harmonic content of $F_{\delta}$ as in $[10,30]$.

\section{Appendix A.4. PM Flux Density Variations}

The pole-cap coefficient $\alpha_{p}$ defined in Figure A1 is an approximate measure of how much flux occurs across a complete pole pitch that is concentrated through the PMs. 
Remark A1. Note that the geometries depicted in Figure A1 are simplified without the presence of eventual magnetic bridges used to fix the PMs. This simplification will render the predicted flux-density magnitudes $B_{v_{m}}$ approximate (mainly due to flux-leakage drop the magnetic bridges). If required, more accurate predictions of $B_{v_{m}}$ can rapidly be obtained using two-dimensional static FEM simulations.

Now, $\psi_{m}\left(\theta_{r}\right)$ (a function of the rotor position $\theta_{r}$ ) is introduced to denote the flux in a PM pole due to $F_{\delta}$. From the geometries depicted in Figure A1, it follows that $\psi_{m}\left(\theta_{r}\right)$ can be approximated as:

$$
\psi_{m}\left(\theta_{r}\right)=\frac{\mu_{0} l_{m} r_{r}}{\left(\delta+h_{m}\right)} \int_{\left(\theta_{r}-\alpha_{p}\right) \pi / p}^{\left(\theta_{r}+\alpha_{p}\right) \pi / p} F_{\delta} d \theta_{s}
$$

where $\mu_{0}$ is the permeability of air. The corresponding flux density in a PM $B_{m}\left(\theta_{r}\right)$ can now be approximated as:

$$
\begin{aligned}
B_{m}\left(\theta_{r}\right) & =\frac{\psi_{m}\left(\theta_{r}\right)}{2 C l_{m} w_{m}} \\
& =\frac{\mu_{0} r_{r}}{2\left(\delta+h_{m}\right) C w_{m}} \int_{\left(\theta_{r}-\alpha_{p}\right) \pi / p}^{\left(\theta_{r}+\alpha_{p}\right) \pi / p} F_{\delta} d \theta_{s}
\end{aligned}
$$

where $C=1$ and $C=1 / 2$ for V-shaped and straight interior PMs, respectively. The harmonic orders present in $B_{m}\left(\theta_{r}\right)$ (of order $v_{m}$ and with a magnitude $B_{v_{m}}$ ) are now obtained from (A11).
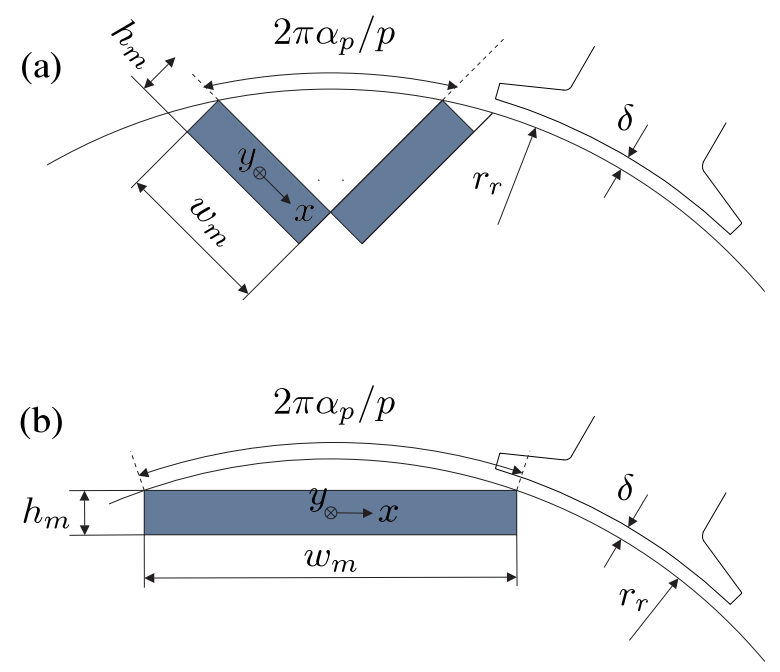

Figure A1. Magnet dimensions and definition of rotor-cap coefficient $\alpha_{p}$ (note that for the coordinate system fixed to PMs, the $y$-axis is directed in the paper): (a) V-shaped interior PMs; (b) straight interior PMs.

Remark A2. When utilizing (A4) and (A5) when inserting (A3) into (A11), it can be verified that the harmonic orders present in the PMs $v_{m}$ can be expressed as:

$$
v_{m}=\left\{\begin{array}{l}
v-p / 2, \text { if } \operatorname{sgn}(v)=1 \text { and } v \neq k p / \alpha_{p} \\
v+p / 2, \text { if } \operatorname{sgn}(v)=-1 \text { and } v \neq k p / \alpha_{p}
\end{array}\right.
$$

where $k$ is an integer. 


\section{Appendix B. IPM Parameters}

Table A1. IPM parameters.

\begin{tabular}{ccc}
\hline Parameter & Value & Unit \\
\hline \# poles $(p)$ & 8 & - \\
\# stator slots $\left(Q_{s}\right)$ & 12 & - \\
\# turns per slot $\left(n_{s}\right)$ & 16 & - \\
Rated current $(I)$ & 97 & $\mathrm{~A}(\mathrm{rms})$ \\
Rotor radius $\left(r_{r}\right)$ & 69.25 & $\mathrm{~mm}$ \\
Air-gap length $(\delta)$ & 0.75 & $\mathrm{~mm}$ \\
Magnet height $\left(h_{m}\right)$ & 7.51 & $\mathrm{~mm}$ \\
Magnet conductivity $\left(\sigma_{m}\right)$ & 694 & $\mathrm{kS} / \mathrm{m}$ \\
Magnet relative permeability $\left(\mu_{r}\right)$ & 1.04 & - \\
\hline
\end{tabular}

\section{References}

1. Gu, W.W.; Zhu, X.Y.; Li, Q.; Yi, D. Design and optimization of permanent magnet brushless machines for electric vehicle applications. Energies 2015, 8, 13996-14008.

2. Lim, S.; Min, S.; Hong, J.-P. Optimal rotor design of IPM motor for improving torque performance considering thermal demagnetization of magnet. IEEE Trans. Magn. 2015, 51, 1-5.

3. Ahn, K.; Bayrak, A.E.; Papalambros, P.Y. Electric vehicle design optimization: Integration of a high-fidelity interior-permanent-magnet motor model. IEEE Trans. Veh. Technol. 2015, 9, 3870-3877.

4. Kato, T.; Minowa, M.; Hijikata, H.; Akatsu, K.; Lorenz, R.D. Design methodology for variable leakage flux IPM for automobile traction drives. IEEE Trans. Ind. Appl. 2015, 51, 3811-3821.

5. Wallscheid, O.; Böcker, J. Global identification of a low-order lumped-parameter thermal network for permanent magnet synchronous motors. IEEE Trans. Energy Convers. 2016, 31, 354-365.

6. EL-Refaie, A.M.; Jahns, T.M. Application of bi-state magnetic material to an automotive IPM starter/ alternator machine. IEEE Trans. Energy Convers. 2005, 20, 71-79.

7. Yang, Z.; Brown, I.P.; Krishnamurtht, M. Comparative study of interior permanent magnet, induction, and switched reluctance motor drives for EV and HEV applications. IEEE Trans. Transport. Electr. 2015, 1, 245-254.

8. Bianchi, N.; Dai Pré, M. Use of the star of slots in designing fractional-slot single-layer synchronous motors. IET Electr. Power Appl. 2006, 153, 459-466.

9. Skaar, S.E.; Krøvel, Ø.; Nilssen, R. Distribution, coil-span and winding factors for PM machines with concentrated windings. In Proceedings of the International Conference on Electrical Machines (ICEM), Chania, Greece, 2-5 September 2006; p. 346.

10. Bianchi, N.; Bolognani, S.; Dai Pré, M.; Grezzani, G. Design considerations for fractional-slot winding configurations of synchronous machines. IEEE Trans. Ind. Appl. 2006, 42, 997-1006.

11. Aslan, B.; Semail, E.; Korecki, J.; Legranger, J. Slot/pole combinations choice for concentrated multiphase machines dedicated to mild-hybrid applications. In Proceedings of the IEEE Industrial Electronics Society (IECON), Melbourne, Australia, 7-10 November 2011; pp. 3698-3703.

12. Bianchi, N.; Fornasiero, E. Index of rotor losses in three-phase fractional-slot permanent magnet machines. IET Electr. Power Appl. 2009, 3, 381-388.

13. Zheng, P.; Wu, F.; Lei, Y.; Sui, Y.; Yu, B. Investigation of a novel 24-slot/14-pole six-phase fault-tolerant modular permanent-magnet in-wheel motor for electric vehicles. Energies 2013, 6, 4980-5002.

14. Bianchi, N.; Bolognani, S.; Fornasiero, E. An overview of rotor losses determination in three-phase fractional-slot PM machines. IEEE Trans. Ind. Appl. 2010, 46, 2338-2345.

15. Tangudu, J.; Jahns, T. Comparison of interior PM machines with concentrated and distributed stator windings for traction applications. In Proceedings of the IEEE Vehicle Power and Propulsion Conference (VPPC), Chicago, IL, USA, 6-9 September 2011; pp. 1-8.

16. EL-Refaie, A.M.; Bolognani, S.; Fornasiero, E. Fractional-slot concentrated-windings synchronous permanent magnet machines: Opportunities and challenges. IEEE Trans. Ind. Electron. 2010, 57, 107-121. 
17. Malloy, A.C.; Martinez-Botas, R.F.; Lampérth, M. Measurement of magnet losses in a surface mounted permanent magnet synchronous machine. IEEE Trans. Energy Convers. 2015, 30, 323-330.

18. Zhang, P.; Sizov, G.Y.; He, J.; Ionel, D.M.; Demerdash, N.O.A. Calculation of magnet losses in concentrated winding permanent magnet synchronous machines using a computationally efficient finite element method. IEEE Trans. Ind. Appl. 2013, 49, 2524-2532.

19. Aslan, B.; Semail, E.; Legranger, J. General analytical model of magnet average eddy-current volume losses for comparison of multiphase PM machines with concentrated winding. IEEE Trans. Energy Convers. 2014, 29, 72-83.

20. Paradkar, M.; Böcker, J. 3D analytical model for estimation of eddy-current losses in the magnets of IPM machine considering the reaction field of the induced eddy currents. In Proceedings of the IEEE Energy Conversion Congress and Exposition (ECCE), Montreal, QC, Canada, 20-24 September 2015; pp. $2862-2869$.

21. Yamazaki, K.; Fukushima,Y. Effect of eddy-current loss reduction by magnet segmentation in synchronous motors with concentrated windings. IEEE Trans. Energy Convers. 2011, 47, 779-788.

22. Chalmers, B.J. Electromagnetic Problems of A.C. Machines; Chapman and Hall: London, UK, 1965; pp. 66-87.

23. Sadarangani, C. Electrical Machines-Design and Analysis of Induction and Permanent Magnet Motors; Universitetesservice: Stockholm, Sweden, 2006; pp. 543-658.

24. Ruoho, S.; Santa-Nokki, T.; Kolehmainen, J.; Arkkio, A. Modeling magnet length in 2-D finite-element analysis of electric machines. IEEE Trans. Magn. 2009, 45, 3114-3120.

25. Krakowski, M. Eddy-current losses in thin circular and rectangular plates. Archiv für Elektrotechnik 1982, 64, 307-311.

26. Sinha, G.; Prabhu, S.S. Analytical model for estimation of eddy current and power loss in conducting plate and its application. Phys. Rev. Spec. Top. Accel. Beams 2011, 14, 062401.

27. Mukerji, S.K.; George, M.; Ramamurthy, M.B.; Asaduzzaman, K. Eddy currents in solid rectangular cores. Prog. Electromagn. Res. B 2008, 7, 117-131.

28. Nategh, S.; Wallmark, O.; Leksell, M.; Zhao, S. Thermal analysis of a PMaSRM using partial FEA and lumped parameter modeling. IEEE Trans. Energy Convers. 2012, 27, 477-488.

29. Zhang, H.; Wallmark, O.; Leksell, M.; Norrga, S.; Harnefors, M.N.; Jin, L. Machine design considerations for an MHF/SPB-converter based electric drive. In Proceedings of the IEEE Industrial Electronics Society (IECON), Dallas, TX, USA, 29 October-1 November 2014; pp. 3849-3854.

30. Mayer, J.; Dajaku, G.; Gerling, D. Mathematical optimization of the MMF-function and -spectrum in concentrated winding machines. In Proceedings of the International Conference on Electrical Machines and Systems (ICEMS), Beijing, China, 20-23 August 2011.

31. Zhao, L.; Li, G. The analysis of multiphase symmetrical motor winding MMF. Electr. Eng. Autom. 2013, 2, $11-18$.

(C) 2017 by the authors. Licensee MDPI, Basel, Switzerland. This article is an open access article distributed under the terms and conditions of the Creative Commons Attribution (CC BY) license (http://creativecommons.org/licenses/by/4.0/). 